\title{
Rol del óxido nítrico en la hipótesis vascular de la enfermedad de Alzheimer
}

Role of nitric oxide in the vascular hypothesis of Alzheimer's disease

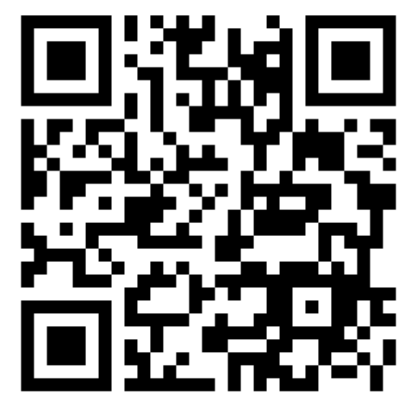

Recibido 05/04/2021

\author{
${ }^{1}$ Dr. Sebastián Fernández Agudelo \\ Investigador independiente, San José, Costa Rica \\ (iD) https://orcid.org/0000-0002-3871-6827 \\ ${ }^{2}$ Dra. Nathalya Zeledón Corrales \\ Área de Salud Oreamuno, San José, Costa Rica \\ (iD) https://orcid.org/0000-0001-9540-1840 \\ ${ }^{3}$ Dr. José Antonio Serrano Suárez \\ Investigador independiente, San José, Costa Rica \\ (iD https://orcid.org/0000-0003-0304-1986
}

Corregido 08/06/2021
Aceptado 20/06/2021

\section{RESUMEN}

El óxido nítrico cumple un papel fundamental en la hipótesis vascular de la enfermedad de Alzheimer. Los tres subtipos de la enzima óxido nítrico sintetasa, encargada de la producción del óxido nítrico en el cuerpo, se ven involucrados en la fisiopatología de esta enfermedad. Primero, la disminución del flujo sanguíneo vascular disminuye la activación de la enzima óxido nítrico sintetasa endotelial. Segundo, la inflamación crónica producto de la formación de placas seniles y la activación de la microglia activa la enzima óxido nítrico sintetasa inducible. Tercero, las alteraciones en los receptores de calcio aumentan la activación de la enzima óxido nítrico sintetasa neuronal. El óxido nítrico también está involucrado de forma directa ya que lleva a un aumento del estrés oxidativo/nitro-oxidativo que altera la función mitocondrial y lleva a la eventual apoptosis de las células de la unidad neurovascular causando el deterioro cognitivo y funcional progresivo propio de la enfermedad de Alzheimer.

PALABRAS CLAVE: enfermedad de Alzheimer; óxido nítrico; estrés oxidativo.

\section{ABSTRACT}

Nitric oxide plays a fundamental role in the vascular hypothesis of Alzheimer's disease. The three subtypes of the enzyme nitric oxide synthase, responsible for the production of nitric oxide in the body, are involved in the pathophysiology of this disease. First, the decrease in vascular blood flow decreases the activation of the endothelial nitric oxide synthetase enzyme. Second, chronic inflammation resulting from senile plaque formation and microglial activation activates 


\begin{abstract}
the inducible nitric oxide synthase enzyme. Third, alterations in calcium receptors increase the activation of the neuronal nitric oxide synthetase enzyme. Nitric oxide is also directly involved as it leads to an increase in oxidative / nitro-oxidative stress that alters mitochondrial function and leads to the eventual apoptosis of the cells of the neurovascular unit, causing the progressive cognitive and functional deterioration typical of the Alzheimer disease.
\end{abstract}

KEYWORDS: Alzheimer's disease; nitric oxide; oxidative stress.

\footnotetext{
${ }^{1}$ Médico general, graduado de la Universidad de Ciencias Médicas (UCIMED). Cód. MED15221. Correo: sebastian.ferdandez.cr.1982@gmail.com

${ }^{2}$ Médica general, graduada de la Universidad de Ciencias Médicas (UCIMED). Cód. MED15226. Correo: nathaliazaledon30@gmail.com

${ }^{3}$ Médico general, graduado de la Universidad de Ciencias Médicas (UCIMED). Cód. MED15665. Correo: jose serrano07@hotmail.com
}

\section{INTRODUCCIÓN}

La enfermedad de Alzheimer (EA) es un proceso neurodegenerativo asociado al envejecimiento y caracterizada por una pérdida progresiva de la memoria, cambios en la personalidad y un deterioro cognitivo del paciente $(1,2)$. La EA es la causa más común de demencia en los adultos mayores a nivel mundial y con el aumento de la expectativa de vida a nivel mundial, la EA se volverá un problema de salud pública ya que se ha descrito que un $45 \%$ de las personas mayores de 85 años padecerán la enfermedad $(3,4)$.

Históricamente, los componentes fisiopatológicos que más peso han tenido en la EA han sido la toxicidad neuronal producida por la acumulación de péptido beta amiloide en placas seniles y la formación de ovillos neurofibrilares como consecuencia de la hiperfosforilación de la proteína tau intraneuronal y la pérdida del esqueleto celular de la neurona $(2,4)$. Por muchos años la investigación sobre la EA se basó en estos procesos fisiopatológicos, con el objetivo de buscar medicamentos y terapias eficaces sin embargo estas terapias no han sido exitosas.

En los últimos años ha habido un cambio de pensamiento sobre la fisiopatología de la EA $y$ se ha visto que esta presenta una combinación de múltiples procesos patológicos complejos. Una de las teorías que más peso ha ganado en las últimas décadas es la hipótesis vascular $(3,4)$. Esta hipótesis une la disfunción endotelial, la hipoperfusión cerebral y la formación de placas seniles y de ovillos neurofibrilares en un solo proceso fisiopatológico que ayuda a esclarecer la compleja y multifactorial patología de la EA lo que permitiría desarrollar terapias más efectivas que las utilizadas actualmente.

La hipótesis vascular está estrechamente ligada a las sustancias que son capaces de controlar el tono vascular de los lechos vasculares cerebrales y que forman parte de la unidad neurovascular. Esta unidad neurovascular está formada por las células endoteliales, las células del musculo liso, los pericitos, las neuronas, los astrocitos y la microglia (5). Esta estrecha relación celular en el sistema nervioso central tiene varios factores en común entre los cuales encontramos al óxido nítrico.

El óxido nítrico por años se estudió en cada una de estas células por solitario, pero hasta los últimos años se ha intentado esclarecer el rol que tiene esta molécula en la estrecha relación de las células de la unión neurovascular.

El objetivo de esta revisión es investigar las últimas investigaciones para comprender la función del óxido nítrico en la fisiopatología de la EA.

\title{
MÉTODO
}


La búsqueda y localización de la información se realizó en las bases de datos PubMed y Scielo. Se escogieron artículos entre el 2010 y el 2019. Se utilizaron los siguientes descriptores: óxido nítrico, EA, disfunción endotelial, vasodilatación cerebral, alteraciones neurovasculares. La búsqueda bibliográfica incluyo artículos científicos de revisión y de resultados experimentales. Para la selección de las publicaciones se analizó el texto completo y se identificaron los más relevantes para desarrollar el artículo. Al final del proceso de selección se escogieron 16 artículos.

\section{HIPÓTESIS VASCULAR DE LA ENFERMEDAD DE ALZHEIMER}

La hipótesis vascular indica que la acumulación patológica de péptido beta amiloide es secundaria al daño vascular (2). Esta hipótesis dice que el primer paso de la EA es una alteración del flujo sanguíneo cerebral (FSC) mediada por factores genéticos, vasculares y ambientales. Esta alteración provoca cambios en las células de la unidad neurovascular que eventualmente provoca hipoperfusión, hipoxia y daño endotelial (2).

Estos cambios vasculares a su vez activan una serie de alteraciones en el sistema nervioso central entre las cuales están el aumento de la producción y la disminución de la depuración del péptido beta amiloide, la hiperfosforilación de la proteína tau y el aumento de mediadores inflamatorios entre otros que al final provocan daño neuronal y un deterioro cognitivo en el paciente $(2,6)$.

\section{GENERALIDADES DEL ÓXIDO NÍTRICO}

El óxido nítrico es un radical libre endógeno que se sintetiza en estado gaseosos y pertenece a la familia de gasotransmisores. Entre las peculiaridades del óxido nítrico está el hecho de que puede traspasar pasivamente la membrana celular para activar directamente la guanilato ciclasa que se encuentra en el espacio intracelular (7). La guanilato ciclasa en una enzima que ayuda en la conversión de guanosín trifostato (GTP) a guanosín monofosfato cíclico (GMPc). Este paso a GMPc activa a su vez la proteína quinasa $G$ la cual a su vez activa la miosina fosfatasa que se encarga de desfosforilar la cabeza de la miosina desacoplando el complejo actina miosina y provocando la vasodilatación de los vasos sanguíneos.

La molécula de óxido nítrico es el principal mecanismo vasodilatador derivado del endotelio. En la circulación cerebral están descritas varias enzimas productoras de óxido nítrico; la óxido nítrico sintetasa endotelial (eNOS) y la óxido nítrico sintetasa neuronal (nNOS) actúan en los procesos fisiológicos de la circulación cerebral y la óxido nítrico sintetasa inducible (iNOS) que es producida por los astrocitos y la microglia como un primer paso del sistema inmune cuando se detecta algún tipo de daño al tejido nervioso $(5,7,8)$.

El óxido nítrico también juega un papel importante en la regulación de la actividad vasoconstrictora y vasodilatadora de los eicosanoides. El balance entre los dos efectos vasculares esta entre otras cosas mediado por la biodisponibilidad de óxido nítrico, los niveles de adenosina, el oxígeno tisular, el lactato y el tono arterial basal (911).

La deficiencia de óxido nítrico promueve diferentes reacciones en el lecho vascular como la vasoconstricción, la agregación plaquetaria, la proliferación de musculo liso y la interacción leucocitaria con el endotelio. Así mismo, en modelos animales con inactivación de la eNOS se ha visto que produce hipertensión, ateroesclerosis e ictus cerebral por lo que el óxido nítrico es un factor común entre los factores de riesgo cardiovasculares y la EA (8). 


\section{ALTERACIONES DE LA eNOS}

En los últimos años se ha intentado esclarecer el rol del óxido nítrico endotelial en la EA. Se han llevado a cabo varios experimentos con modelos animales donde se inhibe la expresión de la eNOS. Entre los hallazgos encontrados se ha visto un aumento en la expresión de la proteína precursora amiloidea (APP) y de la enzima crítica beta-secretasa1 (BACE-1). La BACE1, comúnmente llamada beta secretasa, promueve el proceso amiloidogenico de la APP creando eventualmente las placas seniles $(8,12)$. Al contrario, el GMPc y la snitrosilación del óxido nítrico han demostrado inhibir la BACE-1 disminuyendo la formación de placas seniles y de agregación endotelial de péptido beta amiloide (8).

La disminución del óxido nítrico endotelial también causa una hipoperfusión cerebral como consecuencia de la vasoconstricción no regulada. Esta hipoperfusión va a disminuir el aclaramiento de péptido beta amiloide lo cual va a favorecer la agregación de este péptido llevando a la formación de placas seniles y la consecuente inflamación por parte de la microglia $(7,12)$. Cuando la hipoperfusión cerebral lleva a un estado de hipoxia se activa el factor inducido por hipoxia-1a y este a su vez promueve la acción del BACE-1. Esto lleva a un círculo vicioso en el cual se produce más péptido beta amiloide (13).

En un estudio llevado a cabo por Toda y Okamura se demostró que al exponer arterias basilares de ratas a diferentes precursores del péptido beta amiloide por 6 horas se disminuyó la respuesta dilatadora endotelial y la fosforilación basal de eNOS. Esta información sugiere que en efecto, el péptido beta amiloide inhibe la producción de óxido nítrico endotelial (13).

El óxido nítrico endotelial juega un papel importante en la regulación del tono vascular al contrarrestar inicialmente el efecto de la disminución del flujo sanguíneo cerebral. Sin embargo, cuando el óxido nítrico endotelial disminuye conforme avanza la EA se produce una disfunción de la barrera hematoencefalica, un aumento en el estrés oxidativo y un aumento del péptido beta amiloide agravando la EA y provocando cada vez más síntomas (12). Además de la disminución de óxido nitrosos endotelial, el efecto de la vasoconstricción y la cada vez mayor inflamación crónica contribuyen al ciclo vicioso de la EA.

El FSC en condiciones fisiológicas está controlado por un delicado sistema de balance que media la actividad vasodilatadora y vasoconstrictora de la circulación cerebral. Entre las diferentes vías que actúan sobre la vasodilatación del lecho vascular está el óxido nítrico endotelial y neuronal, principal modulador de la vasodilatación arteriolar. Otras moléculas involucradas en la vasodilatación son el ATP y la adenosina que actúan a través de los receptores $\mathrm{P} 2 Y$. También actúan el metabolito del ácido araquidónico, prostaglandina E2 (PEG2) y el factor hiperpolarizante derivado del endotelio (EDHF). Por otro lado, entre los factores que causan vasoconstricción arteriolar encontramos el ATP y la adenosina a través de los receptores $\mathrm{P} 2 \mathrm{X}$ y el metabolito del ácido araquidónico, el ácido 20hidroxieicosatetraenoico (20-HETE) (6).

En la población adulta el FSC disminuye causando una hipoperfusión cerebral (1). La hipoperfusión causa una acumulación de colágeno en el lecho vascular con un aumento del tamaño de la pared vascular que a su vez dificulta el transporte de oxígeno y nutrientes a las células de sistema nervioso central. Esto a su vez causa daño cerebral que exacerba el deterioro cognitivo de los pacientes con EA $(7,13)$. Cuando disminuye la irrigación sanguínea en el cerebro también aumenta la producción de péptido beta amiloide en las neuronas y 
disminuye la depuración intraneuronal y extraneuronal de la misma (1).

La hipoperfusión cerebral también provoca el depósito de péptido beta amiloide en el lecho vascular provocando una disfunción endotelial y una disminución en la producción de óxido nítrico endotelial (13). Esta acumulación de péptido beta amiloide es llamada angiopatía amiloide cerebral (AAC). La AAC aparte de causar una disfunción endotelial también tiene un efecto citotóxico en las células vasculares causando una pérdida de musculo liso vascular. También se ha relacionado con una mayor predisposición a presentar hemorragias intracraneales y se ha asociado con la disfunción y posterior apoptosis mitocondrial (14).

La AAC también produce una variedad de anomalías vasculares que participan en la progresión de la EA. Entre estas anomalías podemos encontrar vasculitis, perdida de musculo liso vascular y disfunción de la barrera hematoencefalica (15). Sin embargo, en modelos animales se ha visto que estas anomalías se producen aun antes de que se manifieste la AAC por lo que podemos suponer que la AAC exacerba estas patologías, pero no es necesario para que aparezcan en primer lugar.

En modelos animales de ratones con un FSC disminuido y con la expresión de eNOS inhibida, el ejercicio físico no presentó ninguna mejora en el FSC sin embargo en ratones con la expresión de eNOS conservada, el ejercicio físico si aumento el FSC con un subsecuente aumento en el óxido nítrico endotelial y además los ratones presentaron un mejoría cognitiva y funcional (8).

\section{ALTERACIONES DE LA INOS}

El principal rol de las células gliales consta en detectar y activar la respuesta humoral ante cualquier estimulo perjudicial, ya sea un patógeno o un acumulo de proteína beta amiloide, por ejemplo. Una de las moléculas producida durante esta reacción es el óxido nítrico. Este óxido nítrico está producido por niveles elevados de iNOS (7). Durante los procesos inflamatorios propios de la EA se encuentra una sobreexpresión del iNOS que a su vez va a causar una síntesis sostenida de óxido nítrico. El iNOS también se encuentra sobre expresado en ratones envejecidos (5) lo cual sugiere que el aumento de óxido nítrico por la iNOS es de origen multifactorial en la EA.

El óxido nítrico bajo condiciones inflamatorias produce un aumento del estrés oxidativo que a su vez aumenta la formación de placas seniles que provocan más inflamación a nivel cerebral con un mayor aumento en la producción de óxido nitroso. Este círculo vicioso está asociado a la naturaleza degenerativa de la EA (7).

Otro hallazgo importante en modelos animales donde se inhibe la eNOS es el aumento de la molécula adaptadora de calcio ionizado 1 (lba1), el CD68 y el complejo mayor de histocompatibilidad II en la microglia. Estas moléculas están sobre expresadas en la microglia cuando hay procesos inflamatorios activos lo cual sugiere que la disminución del óxido nítrico endotelial propio de la EA contribuye a la activación humoral de la microglia (8).

El óxido nítrico endotelial posee una serie de propiedades inflamatorias que contrarrestan el efecto de la microglia, sin embargo, cuando en la EA disminuye el óxido nítrico endotelial se logra el efecto contrario y se activa la respuesta humoral e inflamatoria por parte de la microglia lo cual a su vez lleva a un aumento de la iNOS (8).

\section{ALTERACIONES DEL nNOS}

En la EA existe un aumento en la expresión de los receptores de rianodina desde las fases iniciales de la enfermedad. Esto causa un aumento en la liberación de calcio intracelular inducida por el calcio que a su 
vez provoca una activación de la nNOS y un aumento en la producción del óxido nítrico neuronal. Esto causa una vasoconstricción que agrava el problema de la hipoperfusión cerebral. El aumento en los niveles de calcio intracelular también se ha relacionado con el estrés oxidativo y nitro-oxidativo que a su vez llevan al daño mitocondrial y la eventual muerte neuronal (7).

El péptido beta amiloide es capaz de alterar la producción de óxido nítrico endotelial al causar un desbalance del calcio intracelular. Se piensa que este desbalance es producto de una fuga de calcio por el receptor de inositol 1,4,5-trisfosfato (IP3) en el retículo endoplasmático (12).

\section{ÓXIDO NITTRICO, ESTRÉS OXIDATIVO Y NITRO-OXIDATIVO}

El estrés oxidativo es un desbalance entre la producción y la eliminación de especies reactivas del oxígeno (EROs) dentro del organismo. Este es producido por las sustancias derivadas del envejecimiento de las mitocondrias en las células del sistema nerviosos y contribuye a la patogénesis de la EA (10). En el cuerpo humano los EROs son producidos por diversas células como las neuronas, la microglia y el endotelio vascular ante ciertos estímulos nocivos (14). Las mitocondrias envejecidas de estas células producen superóxido (O2-) e inhiben ciertas vías antioxidantes como el factor de trascripción Nrf2.

Los EROs son una de las principales causas de disfunción endotelial ya que estos reaccionan con el óxido nítrico para formar peroxinitrito (ONOO-) y ácido peroxinitroso $(\mathrm{ONOOH})$, esto a su vez lleva a un descenso en la biodisponibilidad del óxido nítrico en los vasos cerebrales (9). En modelos experimentales de ratones con sobreexpresión de la APP se han encontrado niveles elevados de peroxinitrito. En estos ratones se encontró que esta formación de peroxinitrito favorecía el estrés nitro-oxidativo $(5,10)$. Este se manifiesta cuando el peroxinitrito reacciona con los ácidos grasos de la membrana y forma varios compuestos entre los que se encuentra el 4-hidroxinonenal (HNE), el cual reacciona con el péptido beta amiloide y lo vuelve más propenso a agregarse y a formar las placas seniles propias de la EA (7).

El peroxinitrito también actúa a nivel mitocondrial ya que causa la nitrosilación de los tioles de las proteínas de membrana formando los llamados tionitritos. Este cambio en la configuración de las proteínas de membrana produce la formación de poros en la membrana mitocondrial y compromete su función permitiendo la salida de compuestos tóxicos como el factor inductor de la apoptosis. Este al salir al citosol induce a la apoptosis neuronal, exacerbando la perdida neuronal encontrada en la EA. El peroxinitrito también actúa sobre la proteína relacionada con la dinamina-1 (Drp-1), una GTPasa que regula la fisión mitocondrial. Cuando el peroxinitrito nitrosila esta proteína se produce una fragmentación mitocondrial, un proceso que lleva a la apoptosis mitocondrial. Esto a su vez causa que las neuronas disminuyan su actividad sináptica y que haya un deterioro cognitivo en los pacientes con EA $(7,16)$.

A nivel mitocondrial el ON actúa compitiendo con el oxígeno para actuar sobre la proteína citocromo c oxidasa. Cuando los niveles de óxido nítrico están aumentados se produce una disfunción en la función de las mitocondrias disminuyendo la energía producida por estas. Este proceso eventualmente lleva también a un aumento de los EROs y a la muerte neuronal (7).

El estrés oxidativo en el lecho vascular también causa una elevada permeabilidad vascular con un aumento de la actividad leucocitaria con una subsecuente inflamación. Esta inflamación causa un aumento de la muerte de células endoteliales que agrava el descenso en la producción de óxido nítrico endotelial (14). 


\section{CONCLUSIONES}

El óxido nítrico es una molécula indispensable en el funcionamiento normal de la unidad neurovascular. Esta se produce a nivel endotelial y neuronal para permitir un adecuado flujo sanguíneo a las regiones cerebrales permitiendo el suministro de nutrientes, oxígeno y una vía de depuración de sustancias tóxicas.

La hipótesis vascular logra unificar muchas de las hipótesis sobre la fisiopatología de la EA y a su vez une los factores de riesgo cardiovasculares con los de la EA. Al ver el protagonismo del lecho vascular en la EA es sencillo ver la importancia del óxido nítrico en las patologías neurodegenerativas. EI óxido nítrico aparte de cumplir su función vasodilatadora influye en muchos otros procesos de la unión neurovascular, ayudando no solo desde el endotelio sino también desde la misma neurona.

El balance del óxido nítrico es un factor crucial en el desarrollo de la EA. Su sobreproducción por las alteraciones de calcio neuronales y la inflamación neurovascular crónica aumenta el estrés oxidativo y nitro-oxidativo causando disfunción mitocondrial severa con una subsecuente apoptosis celular. Por otro lado, su subproducción compromete el flujo sanguíneo cerebral causando una hipoperfusión a los tejidos además de permitir una producción aumentada del péptido beta amiloide que activa el sistema inmune causando una inflamación crónica y llevando a una eventual disfunción endotelial lo que crea un círculo vicioso.

Dada la importancia del delicado balance del óxido nítrico en la unidad neurovascular, se debería de enfocar la investigación actual en terapias que regulen de manera efectiva estas vías para así lograr encontrar una terapia con mejores resultados contra la futura epidemia que será la EA.
Las nuevas terapias deberían enfocarse en reducir la sobreproducción de óxido nítrico por parte de la iNOS y la nNOS y a su vez proteger el endotelio de las sustancias perjudiciales que llevan a una disminución de la producción de óxido nítrico endotelial por la eNOS. De esta manera se lograría acabar con el circulo vicioso de la EA y tal vez detener la progresión acelerada de la enfermedad al mismo tiempo que mejorar las funciones cognitivas de los pacientes con EA.

\section{Los autores declaran no tener conflicto de interés.}

\section{REFERENCIAS}

1. Iadecola C. Vascular and metabolic factors in Alzheimer's diseases and related dementias: Introduction. Cell Mol Neurobiol. marzo de 2016;36(2):151.

2. Lyros E, Bakogiannis C, Liu Y, Fassbender K. Molecular links between endothelial dysfunction and neurodegeneration in Alzheimer's disease. Curr Alzheimer Res. enero de 2014;11(1):18-26.

3. Janota C, Lemere CA, Brito MA. Dissecting the Contribution of Vascular Alterations and Aging to Alzheimer's Disease. Mol Neurobiol. 2016;53(6):3793-811.

4. Yamazaki Y, Kanekiyo T. Blood-Brain Barrier Dysfunction and the Pathogenesis of Alzheimer's Disease. Int $\mathrm{J}$ Mol Sci. 13 de septiembre de 2017;18(9).

5. Lourenço CF, Ledo A, Barbosa RM, Laranjinha J. Neurovascular uncoupling in the triple transgenic model of Alzheimer's disease: Impaired cerebral blood flow response to neuronal-derived nitric oxide signaling. Exp Neurol. 2017;291:36-43.

6. Kisler K, Nelson AR, Montagne A, Zlokovic BV. Cerebral blood flow regulation and neurovascular dysfunction in Alzheimer disease. Nat Rev Neurosci. 18 de mayo de 2017;18:419.

7. Asiimwe N, Yeo SG, Kim M-S, Jung J, Jeong NY. Nitric Oxide: Exploring the Contextual Link with Alzheimer's Disease. Oxid Med Cell Longev. 2016;2016:7205747.

8. Katusic ZS, Austin SA. Neurovascular Protective Function of Endothelial Nitric Oxide - Recent Advances. Circ J Off J Jpn Circ Soc. 24 de junio de 2016;80(7):1499-503.

9. Tarantini S, Tran CHT, Gordon GR, Ungvari Z, Csiszar A. Impaired neurovascular coupling in aging and Alzheimer's disease: Contribution of 
astrocyte dysfunction and endothelial impairment to cognitive decline. Exp Gerontol. 2017;94:52-8.

10. Toth P, Tarantini S, Csiszar A, Ungvari Z. Functional vascular contributions to cognitive impairment and dementia: mechanisms and consequences of cerebral autoregulatory dysfunction, endothelial impairment, and neurovascular uncoupling in aging. Am J Physiol Heart Circ Physiol. 1 de enero de 2017;312(1):H120.

11. Yagami T, Koma $\mathrm{H}$, Yamamoto $\mathrm{Y}$. Pathophysiological Roles of Cyclooxygenases and Prostaglandins in the Central Nervous System. Mol Neurobiol. 2016;53(7):4754-71.

12. Di Marco LY, Venneri A, Farkas E, Evans PC, Marzo A, Frangi AF. Vascular dysfunction in the pathogenesis of Alzheimer's disease--A review of endothelium-mediated mechanisms and ensuing vicious circles. Neurobiol Dis. octubre de 2015;82:593-606.

13. Toda N, Okamura T. Cigarette smoking impairs nitric oxide-mediated cerebral blood flow increase: Implications for Alzheimer's disease. J Pharmacol Sci. agosto de 2016;131(4):223-32.

14. Salmina $A B$, Inzhutova $A I$, Malinovskaya NA, Petrova MM. Endothelial dysfunction and repair in Alzheimer-type neurodegeneration: neuronal and glial control. J Alzheimers Dis JAD. 2010;22(1):1736.

15. Merlini M, Shi Y, Keller S, Savarese G, Akhmedov $A$, Derungs $R$, et al. Reduced nitric oxide bioavailability mediates cerebroarterial dysfunction independent of cerebral amyloid angiopathy in a mouse model of Alzheimer's disease. Am J Physiol Heart Circ Physiol. 1 de febrero de 2017;312(2):H232-8.

16. Wang W, Yin J, Ma X, Zhao F, Siedlak SL, Wang $Z$, et al. Inhibition of mitochondrial fragmentation protects against Alzheimer's disease in rodent model. Hum Mol Genet. 01 de 2017;26(21):411831. 